\title{
BMJ Open Are there gender differences in acute management and secondary prevention of acute coronary syndromes in Barbados? A cohort study
}

\author{
Natasha Sobers, ${ }^{1}$ Angela M C Rose, ${ }^{2}$ T Alafia Samuels, ${ }^{2}$ Julia Critchley, ${ }^{3}$ \\ Melissa Abed, ${ }^{1}$ Ian Hambleton, ${ }^{2}$ Arianne Harvey, ${ }^{1}$ Nigel Unwin ${ }^{2,4}$
}

To cite: Sobers N, Rose AMC, Samuels TA, et al. Are there gender differences in acute management and secondary prevention of acute coronary syndromes in Barbados? A cohort study. BMJ Open 2019;9:e025977. doi:10.1136/ bmjopen-2018-025977

- Prepublication history for this paper is available online. To view these files, please visit the journal online (http://dx.doi. org/10.1136/bmjopen-2018025977).

Received 11 August 2018 Revised 4 December 2018 Accepted 5 December 2018

Check for updates

(C) Author(s) (or their employer(s)) 2019. Re-use permitted under CC BY-NC. No commercial re-use. See rights and permissions. Published by BMJ.

${ }^{1}$ Faculty of Medical Sciences, University of the West Indies, Bridgetown, Barbados ${ }^{2}$ The George Alleyne Chronic Disease Research Centre, Caribbean Institute for Health Research, University of the West Indies, Bridgetown, Barbados ${ }^{3}$ Population Health Research Institute, St George's, University of London, London, UK ${ }^{4}$ MRC Epidemiology Unit, UKCRC Centre for Diet and Activity Research (CEDAR), University of Cambridge School of Clinical Medicine, Cambridge, UK

Correspondence to Dr Natasha Sobers; natasha.sobers@cavehill.uwi. edu

\section{ABSTRACT}

Objectives In Barbados, high case fatality rates have been reported after myocardial infarction (MI) with higher rates in women than men. To explore this inequality, we examined documented pharmacological interventions for ST-segment elevated myocardial infarction (STEMI), non-STEMI (NSTEMI) and unstable and chronic angina in women and men.

Design Prospective cohort registry data for STEMI and NSTEMI and retrospective chart review for unstable and chronic angina.

Setting Tertiary care (acute coronary syndromes) and primary care (chronic angina) centres in Barbados. Participants For the years 2009-2016, a total of 1018 patients with STEMI or NSTEMI were identified via the prospective study. For unstable and chronic angina, 136 and 272 notes were reviewed respectively for the years 2010-2014.

Outcome measures The proportions of patients prescribed recommended medication during the first 24 hours after an acute event, at discharge and for chronic care were calculated. Prescribed proportions were analysed by gender after adjustment for age.

Results Between 2009 and 2016, for the acute management of patients with NSTEMI and STEMI, only two (aspirin and clopidogrel) of six drugs had documented prescription rates of $80 \%$ or more. Patients with STEMI $(n=552)$ had higher prescription rates than NSTEMI $(n=466)$, with gender differences being more pronounced in the former. Among patients with STEMI, after adjustment for age, diabetes, hypertension and smoking, men were more likely to receive fibrinolytics acutely, OR $2.28(95 \% \mathrm{Cl} 1.24$ to 4.21). Compared with men, a higher proportion of women were discharged on all recommended treatments; this was only statistically significant for beta-blockers: age-adjusted OR 1.87 (95\% Cl 1.16 to 3.00 ). There were no statistically significant differences in documented prescription of drugs for chronic angina.

Conclusion Following acute $\mathrm{Ml}$ in Barbados, the proportion of patients with documented recommended treatment is relatively low. Although women were less likely to receive appropriate acute care than men, by discharge gender differences were reversed.

\section{Strengths and limitations of this study}

- Population-based prospectively collected data for all ST-segment elevated myocardial infarctions (STEMI) and non-STEMIs (NSTEMI) from a small island developing state (SIDS).

- Several years of data collection allowing adequate power to detect important gender differences for STEMI and NSTEMI.

- Inadequate power to detect potentially important gender differences for unstable angina.

- Significant barriers to collection of data from paper-based records as occurred in SIDS were overcome.

Only $68 \%$ of notes search for were retrieved for unstable angina in hospital while $87 \%$ of notes in primary care for chronic angina were retrieved.

\section{INTRODUCTION}

Coronary heart disease (CHD) is associated with high mortality prehospital, in-hospital and in the weeks following hospital discharge. ${ }^{1-3}$ The risk of death and future events for patients experiencing an acute coronary syndrome (ACS) can be reduced substantially through the timely introduction of treatment. ${ }^{4}$ Despite this, adherence to guidelines by physicians has not always been adequate. $^{56}$

Globally, gender differences exist with respect to ACS outcomes and treatment. ${ }^{7}$ In Barbados, unpublished data indicate that in-hospital acute myocardial infarction (MI) case fatality rate (2013) was $40.8 \%$ (95\% CI 32.0 to 51.2) with higher rates in women after age adjustment (risk ratio $=1.18 ; 95 \%$ CI 1.02 to 1.37$).{ }^{8}$ The 2016 annual report of the national registry of Barbados indicated that the age-standardised mortality rate for MI was 56.2 per 100000 population. ${ }^{9}$ Debate exists internationally related to whether the difference in outcomes is due to treatment 
bias or biology. ${ }^{10}$ Published treatment data are unavailable for Barbados and the Caribbean. Internationally, some studies have shown women are less likely to receive timely treatment in the acute period and are more likely to receive medical management rather than primary percutaneous coronary intervention $(\mathrm{PCI})^{11-13}$ while others from the USA have shown no difference. ${ }^{7}$ Studies vary considerably in the gender differences observed in treatment given at discharge. ${ }^{11} 14$

Despite knowing that the level of adherence and availability of treatments is critical to reducing cardiovascular mortality, we were unable to find any studies from the Caribbean within the past 25 years which provide a description of treatment used in the management of ACS. In this study, we examine the documented treatment proportion rates for ST-segment elevated myocardial infarction (STEMI) and non-STEMI (NSTEMI) within the first 24 hours as well as at discharge. We have chosen to use class 1 recommendations from the American College of Cardiology/American Heart Association (ACC/AHA) Task Force on Practice Guidelines. ${ }^{15}$ Given the gender difference noted in mortality rates, the main aim of this paper is to explore gender differences in documented prescribing practices for each ACS and evaluate whether patients with ACS in Barbados are treated with optimal medical therapy in both the hospital and community settings.

\section{METHODS}

\section{Study sites and design}

Data for this study were obtained from two main sources-a population-based national registry, and a retrospective chart review conducted in two sites (the country's lone tertiary public sector hospital and the island-wide government-operated primary care centres). The national registry provided information on proportions of patients prescribed specific drugs for acute MI during the first 24 hours of symptoms and on discharge from the main hospital. The retrospective hospital chart review provided information on medications provided to patients with unstable angina, while the primary care section of this review provided data on medications prescribed for patients with chronic angina. Our reporting was guided by Strengthening the Reporting of Observational Studies in Epidemiology guidelines for cohort studies. ${ }^{16}$

\section{MI: population-based data (registry)}

We obtained data for the years 2009-2016 from the Barbados National Registry for Chronic Non-communicable Disease (the BNR), the only multidisease registry in the Western Hemisphere. ${ }^{17}$ This national surveillance system collects data from hospital and community records for all patients diagnosed with an acute MI, using a mixture of active and passive case finding. ${ }^{17}$ After MI events are verified, patient notes are abstracted and later entered into a database for analysis and dissemination. To accomplish its case finding, the BNR engages both the public and private healthcare sectors. One of the main sources of data is the Queen Elizabeth Hospital (QEH) - a 600-bed facility, the island's lone tertiary care centre. Within the QEH, data are provided by the accident and emergency department, medical records and death records departments and the medical and surgical wards. Data sources outside the QEH include the polyclinics (government-operated primary care centres), private physicians, therapists, emergency centres and the National Register of Births and Deaths.

The BNR collects information on medications used acutely within the first 24 hours of onset. In addition, medications given at discharge and chronic medications used by the patient prior to the event are registered. Medication sources include ambulance reports; referral sheets/ letters from emergency clinics and general practitioners (GP) (any medications documented as having been given at the GP/emergency clinic before hospital admission); accident and emergency records sheet; nurses' report; physicians' treatment card; and the patient discharge note.

\section{Unstable angina in the tertiary care centre}

Because the BNR does not collect data on patients with unstable angina, a retrospective chart review was conducted at the QEH. The participant population comprised adult residents of Barbados treated at the hospital for unstable angina. No limits were placed on race, gender or geographic locations within Barbados. Private sector management of ACS was not conducted due to lack of resources.

In the hospital review, we obtained a list of all records with a discharge diagnosis of unstable angina, filed using the International Classification of Diseases-Tenth Revision I20.0 code during years 2010-2014, which totalled 614 patients. To estimate sample size, we assumed all drug proportions at $50 \%$ for the purpose of the power calculation, to produce $95 \%$ CIs of $\pm 7.5 \%$. We estimated we would require 180 notes for review but in anticipation of having irretrievable records we chose to sample 200.

From the list of all unstable angina records, a systematic (every third) sample of 200 records was requested from the list of all persons admitted to hospital, of which 136 (68\% response rate) notes were located. Data were abstracted by trained abstractors and entered into a secure electronic database and files encrypted. Abstracted data were entered into an EPI INFO database. We abstracted only the medications prescribed on discharge using physicians' and nurses' records contained in the patient's paper-based file.

\section{Chronic angina in polyclinics}

As an assessment of community treatment, a retrospective review was conducted within the eight public primary care centres in Barbados to determine the proportions of patients with chronic angina receiving adequate treatment. A records clerk used the polyclinics' electronic medical database system to extract all persons who were 
seen in the polyclinics during the period 2010-2014 with a diagnosis listed as 'Ischaemic Heart Disease-Angina' regardless of whether this was the main reason for the visit. (This is the code for angina in accordance with the International Primary Care Coding system.) All visits of each of these persons during the 5-year period were abstracted into an Excel spreadsheet. After duplicate records/visits were removed, a total of 834 unique individuals were seen in the public sector with this diagnosis in 2010-2014.

A sample of 272 notes were selected using simple random sampling (using an Excel random number generator) from this list and given to the data abstractor for retrieval. Notes were regarded as eligible if documentation confirmed that patients were seen at the clinic at least twice for this condition in the past 12 months. Of the 272 notes, 236 ( $87 \%$ response rate) were found and noted to be eligible for data abstraction. Records could not be found for two persons, who were noted to be deceased. Due to the difficulty in tracing medical records when examining unstable and chronic angina using the chart reviews, results may be less robust than BNR data, thus a greater focus is placed in analysis on acute MI data from the BNR. We present the unstable and chronic angina data, however, as the best available data from our setting.

\section{Data analysis}

Prescribed drugs were recorded and classified by type-statins, beta-blockers (BB), fibrinolytic drugs and ACE inhibitor (ACEI) or angiotensin II receptor blocker (ARB). We summarised categorical variables, including disease status and drugs prescribed, as n (\%) and continuous variables as mean (SD). We compared proportions between groups using $95 \%$ CIs. We calculated the prescribed proportions by age groups (30-69 years old and 70 years and older) and gender for drugs considered recommended by class 1 evidence by ACC/ AHA for post-MI and unstable angina treatment as well as the proportion of patients with chronic angina in the community by age and gender receiving prescriptions for aspirin, clopidogrel, statins, BB and ACEI or ARB. The AHA/ACC recommends aspirin (ASA), P2Y ${ }_{12}$ inhibitor, fibrinolytics and anticoagulation for management of STEMI in the first 24 hours and ASA, any P2Y ${ }_{12}$ inhibitor, BB, statin \pm ACEI or ARB on discharge. Nitrates are also recommended for symptomatic relief only. Since our interest is in the effectiveness of secondary prevention modalities, nitrates and anticoagulation treatment were not analysed.

We assessed the effects of age (measured as a continuous variable) and gender on aspirin, clopidogrel, statins, $\mathrm{BB}$, fibrinolytic drugs and ACEI or ARB using multivariable logistic regression. We conducted bivariate analyses to explore the effects of documenting each of the following comorbidities (hypertension, diabetes, previous ischaemic heart disease, obesity, smoking, history of coronary angiography, atrial fibrillation, congestive cardiac failure and systolic blood pressure on presentation) on fibrinolytic use for patients with STEMI. Using Akaike information criterion, the final model explaining the relationship between reperfusion and predictors of adherence included age, gender, medical history of diabetes, hypertension and smoking. Complete adherence to prescribing was defined as persons discharged on all five recommended medications proven to reduce morbidity and mortality post-CHD event. For those in the community, complete adherence to prescribing was defined as those on the four medications recommended for stable angina. Data were analysed using Stata V.13.

\section{Patient and public engagement}

Patients and the public were not actively involved in this study. Data from these findings have been disseminated to the healthcare providers and key stakeholders to facilitate policy and planning around care of patients with MI in Barbados. Dissemination targeted at the general public was released at a 70th Anniversary Celebration open day of the University of the West Indies in November 2018.

\section{Ethical considerations}

This study was approved by the Institutional Review Board of the University of the West Indies/Ministry of Health, as well as the internal ethics committees of the QEH and the Ministry of Health (the government agency responsible for management of the public primary care centres). No information on patient identifiers was abstracted since participants were not followed or contacted after this audit.

\section{RESULTS}

For the years 2009-2016, there were 2750 patients identified by the BNR with a diagnosis of acute MI. Of these, 1589 were admitted to hospital; 1295 notes were completely reviewed by the registry data abstraction team while 294 were diagnosed based on only their death certificate. For 1018, the diagnosis of acute MI was definitively made using ECG and cardiac enzymes. Classification of these patients according to type of acute MI revealed that $45.8 \%$ (466) were diagnosed with NSTEMI and 54.2\% (552) with STEMI (figure 1).

A higher proportion of men were diagnosed with STEMI compared with women: $58.8 \%$ vs $48.8 \%$, respectively $(\mathrm{p}=0.001)$. Women generally had significantly higher rates of several comorbidities (hypertension, diabetes and obesity). Men had significantly higher rates of smoking (table 1).

\section{Documented medications prescribed in first 24 hours for patients with STEMI/NSTEMI}

Overall in acute management of patients with NSTEMI and STEMI, documentation indicated that only two (aspirin and clopidogrel) of six drugs had documented prescription greater than $80 \%$. Aspirin was the most prescribed drug: $87 \%$ (95\% CI $83 \%$ to $89 \%$ ) with no significant gender or age differences in prescribing 


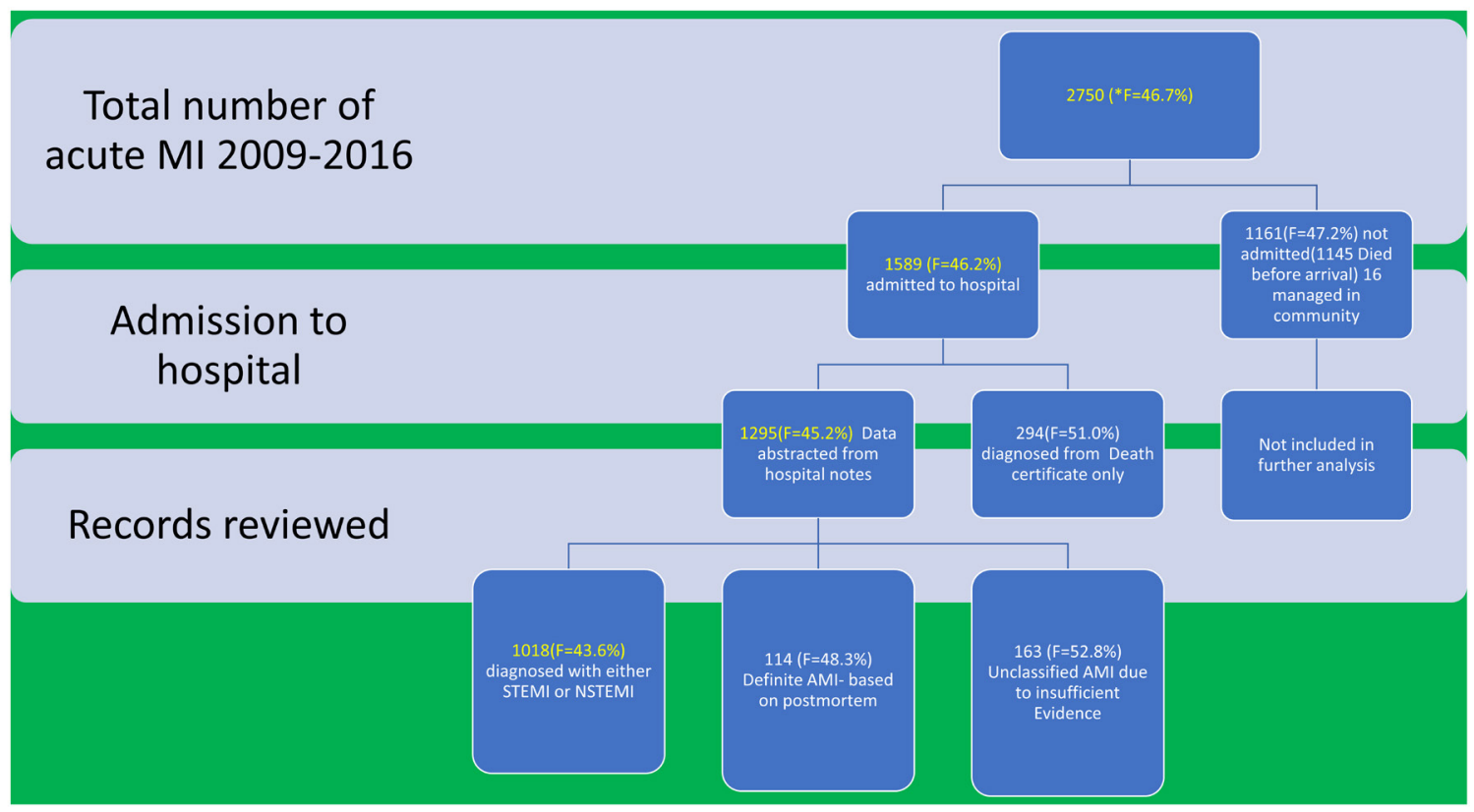

Figure 1 Numbers of eligible patients with AMI for 2009-2016 in Barbados. AMI, acute myocardial infarction; MI, myocardial infarction; NSTEMI, non-STEMI; STEMI, ST-segment elevated myocardial infarction.

proportions, while a fibrinolytic drug was least likely to be prescribed, $57 \%$ (95\% CI $51 \%$ to $63 \%$ ) in men and $31 \%$ (95\% CI $24 \%$ to $39 \%$ ) in women. Figure 2 displays the proportion of documented medications prescribed in younger (30-69 years old) and older (70 years and older) age groups by gender. Persons with NSTEMI had relatively lower prescription than STEMI, with gender significant differences noted for the latter. In patients with STEMI, after adjustment for age, men were more than twice as likely to receive fibrinolytics compared with women: OR
2.38 (95\% CI 1.56 to 3.63 ) and $45 \%$ more likely to receive clopidogrel: OR 1.45 (95\% CI 0.87 to 2.43 ).

After bivariate analysis, smoking was found to be positively associated with fibrinolytic use-crude OR 2.21, 95\% CI (1.27 to 3.83). Persons with a history of diabetes and hypertension were less likely to receive fibrinolysiscrude OR 0.44 (95\% CI 0.27 to 0.72 ) and crude OR 0.59 (95\% CI 0.36 to 0.99 ), respectively. The most parsimonious model showed that age and sex were statistically significant predictors of use of fibrinolytics. Patients with

Table 1 Characteristics of patients with acute myocardial infarction (MI) by gender and MI classification

\begin{tabular}{|c|c|c|c|c|}
\hline & \multicolumn{2}{|l|}{ STEMI } & \multicolumn{2}{|l|}{ NSTEMI } \\
\hline & Male $(n=341)$ & Female $(n=211)$ & Male $(n=234)$ & Female $(n=236)$ \\
\hline Mean age $(95 \% \mathrm{Cl})$ & $61.9(60.4$ to 63.4$)$ & 69.1 (67.3 to 70.8$)$ & $64.5(62.8$ to 66.4$)$ & 70.0 (68.2 to 71.8$)$ \\
\hline Hypertension, \% (95\% Cl) & $74.9(69.7$ to 80.1$)$ & 89.7 (85.4 to 94.0$)$ & 78.9 (73.2 to 84.6$)$ & 93.9 (90.5 to 97.3$)$ \\
\hline $\begin{array}{l}\text { Refractory hypertension } \\
(\mathrm{SBP}>180 \mathrm{~mm} \mathrm{Hg})\end{array}$ & 11.9 (8.3 to 15.4$)$ & $15.6(10.7$ to 20.5$)$ & 16.1 (11.2 to 20.9$)$ & 28.9 (22.9 to 34.9$)$ \\
\hline Angina & 42.9 (32.0 to 52.8$)$ & 60.4 (46.3 to 74.5$)$ & $55.4(42.1$ to 68.6$)$ & 77.0 (66.3 to 87.8$)$ \\
\hline Previous stroke & 13.3 (6.9 to 19.6$)$ & 17.1 (8.5 to 25.7$)$ & 17.6 (8.4 to 26.9$)$ & $21.0(12.0$ to 30.0$)$ \\
\hline Previous CABG & $25.0(12.4$ to 37.6$)$ & 15.0 (0.0 to 31.4$)$ & 21.1 (1.5 to 40.6$)$ & $35.3(11.0$ to 59.5$)$ \\
\hline Atrial fibrillation & 26.2 (15.0 to 37.5$)$ & 35.3 (18.8 to 51.8$)$ & $31.2(14.6$ to 47.9$)$ & $34.3(18.0$ to 50.5$)$ \\
\hline Previous coronary angiography & 10.5 (0.0 to 20.6$)$ & $0.6(0.0$ to 17.7$)$ & $18.7(0.0$ 39.5) & 9.0 (0.0 to 27.8$)$ \\
\hline
\end{tabular}

CABG, coronary artery bypass graft; NSTEMI, non-STEMI; SBP, systolic blood pressure; STEMI, ST-segment elevated myocardial infarction. 


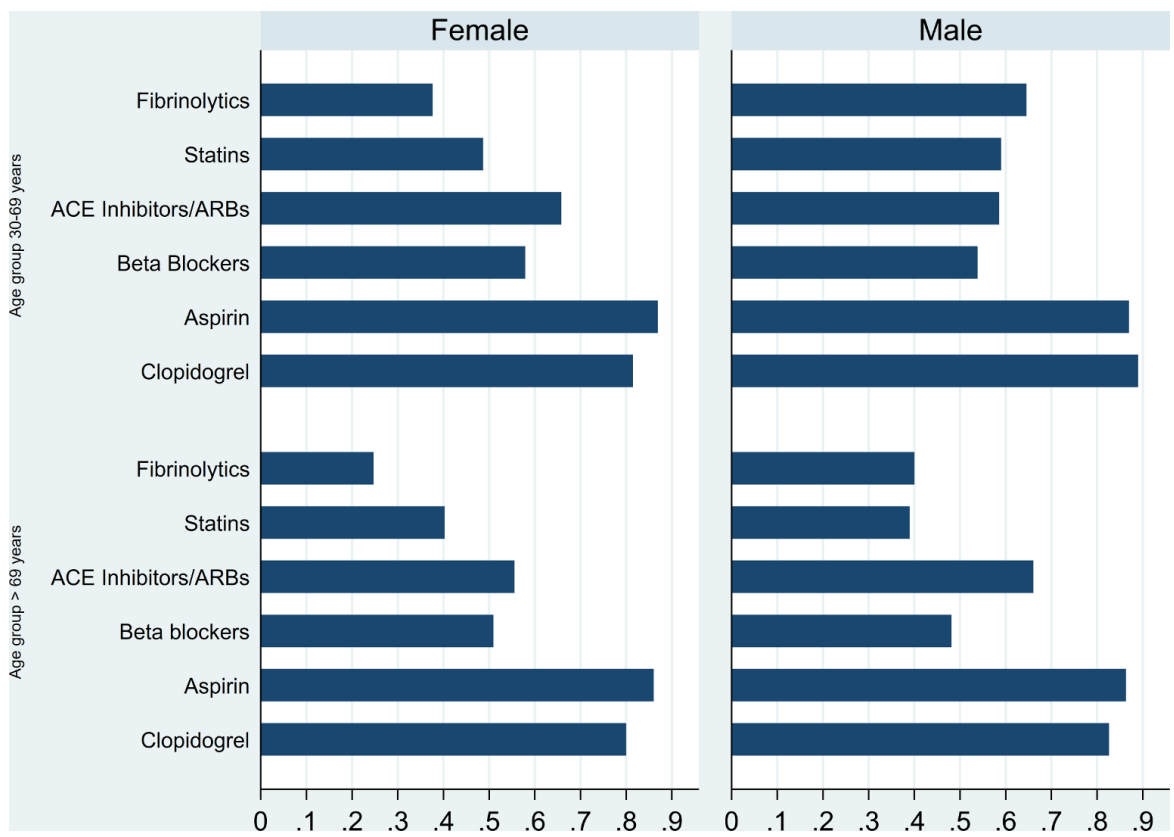

Figure 2 Proportion of patients with ST-segment elevated myocardial infarction (STEMI) who received recommended treatment in the first 24 hours of hospital admission by age and gender. ARB, angiotensin II receptor blocker.

STEMI were less likely (adjusted OR $0.97,95 \%$ CI 0.95 to $0.99)$ to receive fibrinolytics for every 1 year increase in age, while men were more likely (adjusted OR 2.28, $95 \%$ CI 1.24 to 4.21 ) than women to receive fibrinolytic treatment after adjustment for diabetes, hypertension and smoking.

Persons less than 70 years old tended to have documented prescription proportions similar to those over 70 years for clopidogrel, aspirin and BB, but the younger age group was prescribed more aggressive therapy in the case of statins and fibrinolytics. Age (modelled as a continuous variable) was a significant predictor for the use of fibrinolytics, with younger persons being more likely to receive this form of therapy (table 2). After adjustment for age, diabetes, hypertension, smoking and systolic blood pressure on admission, men with STEMI were 2.34 (95\% CI 1.25 to 4.36 ) times more likely to be given reperfusion treatment than their female counterparts.

\section{Discharge trends}

Documented prescribing proportions on discharge ranged from $62 \%$ (95\% CI $57 \%$ to $67 \%$ ) for clopidogrel therapy to $84 \%$ ( $95 \%$ CI $80 \%$ to $88 \%$ ) for BB treatment in patients with STEMI and from $72 \%$ (95\% CI 66\% to 78\%) for clopidogrel to $87 \%$ (95\% CI $82 \%$ to $91 \%$ ) for aspirin in patients with NSTEMI (figure 3). Persons younger than 70 years old had higher documented prescription rates for all drugs except in the case of men on BB and statins. Logistic regression indicated that women and younger persons were generally more likely to be discharged on recommended medications. The exception to this occurred for women on aspirin and BB where older persons had higher documented prescriptions.

Patients with angina in hospital and the community

At the QEH, for the period 2010-2014, 57.2\% (78) of the 138 patients with unstable angina were female. The mean

Table 2 Logistic regression displaying the extent to which gender (crude and age adjusted) predicted proportions of medications prescribed for persons with STEMI/NSTEMI during the first 24 hours

\begin{tabular}{llllll}
\hline & STEMI OR $(\mathbf{9 5 \%} \mathbf{C I})$ & & & NSTEMI OR (95\% CI) \\
\cline { 2 - 3 } \cline { 5 - 6 } Female (reference) & Crude & Adjusted & & Crude & Adjusted \\
\hline Aspirin & $1.02(0.61$ to 1.72$)$ & $0.95(0.56$ to 1.63$)$ & & $1.34(0.79$ to 2.27$)$ & $1.37(0.79$ to 2.35$)$ \\
Clopidogrel & $1.60(0.97$ to 2.63$)$ & $1.45(0.87$ to 2.43$)$ & & $1.19(0.73$ to 1.94$)$ & $1.20(0.73$ to 1.97$)$ \\
Beta-blockers & $0.91(0.65$ to 1.27$)$ & $0.85(0.60$ to 1.20$)$ & & $0.88(0.61$ to 1.26$)$ & $0.83(0.57$ to 1.20$)$ \\
ACE_ARB & $1.00(0.71$ to 1.42$)$ & $0.99(0.70$ to 1.42$)$ & & $1.17(0.81$ to 1.69$)$ & $1.07(0.74$ to 1.57$)$ \\
Statins & $1.40(0.93$ to 2.10$)$ & $1.24(0.82$ to 1.90$)$ & & $1.19(0.73$ to 1.94$)$ & $1.13(0.69$ to 1.86$)$ \\
Fibrinolytics & $2.93(1.95$ to 4.41$)$ & $2.38(1.56$ to 3.63$)$ & NA & NA & \\
\hline
\end{tabular}

ARB, angiotensin II receptor blocker; NA, not applicable; NSTEMI, non-STEMI; STEMI, ST-segment elevated myocardial infarction. 


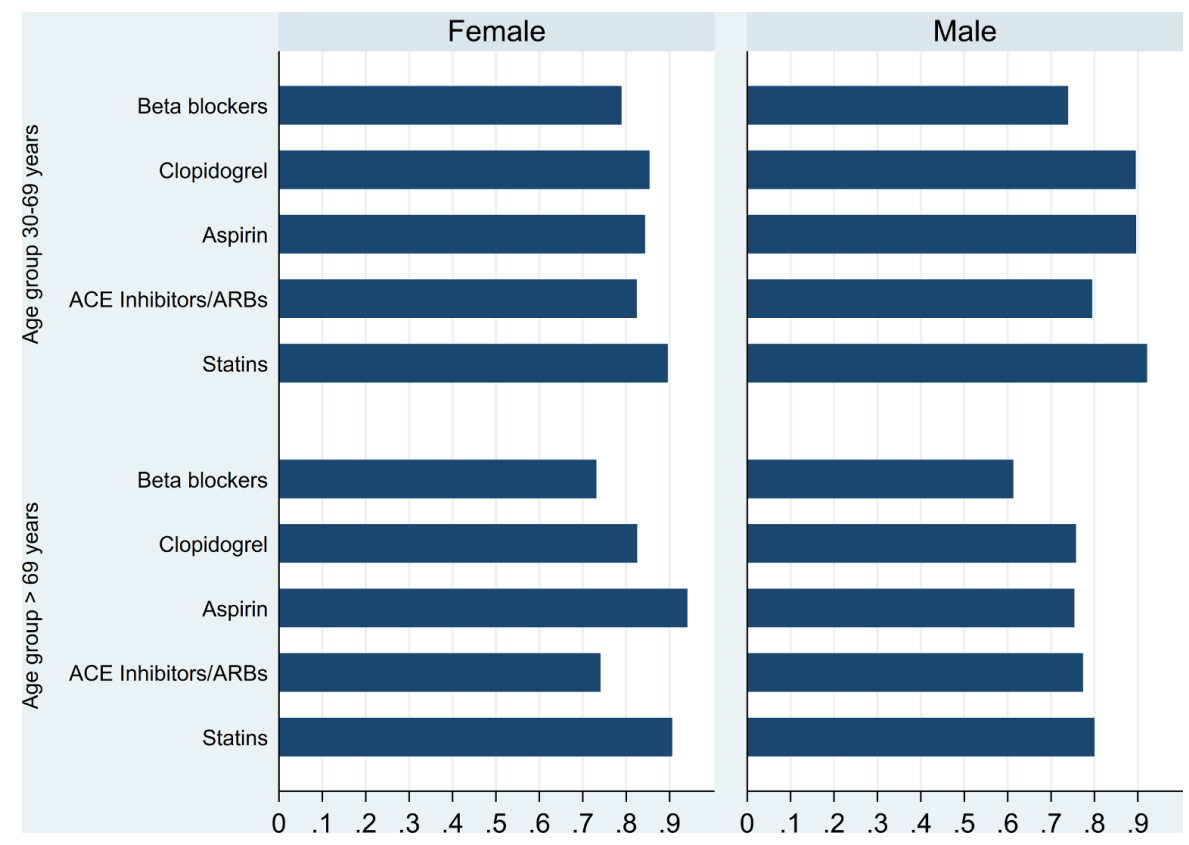

Figure 3 Proportion of patients with ST-segment elevated myocardial infarction (STEMI) who received recommended treatment on discharge from hospital by age and gender. ARB, angiotensin II receptor blocker.

ages for men and women were 65.3 (SD 12.7) and 61.1 (SD 13.5), respectively. Documented prescribing proportions for patients with unstable angina ranged from $57 \%(95 \%$ CI $48 \%$ to $65 \%$ ) (clopidogrel) to $88 \%$ (95\% CI $81 \%$ to $93 \%$ ) (aspirin). There were no significant differences by gender or age.

In the community, the prescribed proportions were generally lower for use of ACEI/ARBs (58\% in men, $63 \%$ in women) and BB (52\% in men and $63 \%$ in women) but relatively high for statins ( $67 \%$ in men and $74 \%$ in women) and aspirin ( $81 \%$ in men and $77 \%$ in women). Older persons were more likely to be treated in all medication and gender groups but none of these differences reached statistical significance.

\section{Hospital versus community physician adherence to recommended medications for patients with CHD}

Documented prescribing adherence to recommended medications was generally higher in hospital compared with the community. In persons diagnosed with STEMI, 36.4\% (95\% CI 29.8 to 0.43 .5 ) were documented as discharged on all five medications recommended, while among patients with NSTEMI, a similar proportion was noted $(35.9 \%$; $95 \%$ CI 30.9 to 41.2). Almost one-third of patients with unstable angina were discharged from hospital with all five medications recommended. This was similar in men and women: $28.3 \%$ (95\% CI $17.4 \%$ to $41.4 \%$ ) vs $33.3 \%$ (95\% CI $23.2 \%$ to $44.7 \%)$, respectively. Age and gender were not significant predictors of complete adherence.

\section{DISCUSSION}

When comparing gender distributions for ACS, several patterns emerged. Men had higher prescription proportions in the first 24 hours of care for all drugs examined except $\mathrm{BB}$, but the difference was significantly higher than women in the case of fibrinolytics used for reperfusion therapy after adjustment for age and other comorbidities. This gender difference in the first 24 hours may explain the higher case fatality seen in women in Barbados. Similar findings were noted in Puerto Rico and Australia. ${ }^{1819}$ The absence of primary percutaneous intervention during this time period may also be a contributor to the relatively high case fatality rates in both sexes.

Documented prescription rates in the first 24 hours in Barbados were lower than those reported in USA and Canadian-based registries ${ }^{20}{ }^{21}$ and in an analysis of the Australian and New Zealand cohort of the Global Registry of Acute Coronary Events (GRACE). ${ }^{22}$ The discharge prescription rates were similar: proportions of medications prescribed were $95 \%, 85 \%$ and $89 \%$ for aspirin, BB and statin use, respectively, in the GRACE study. ${ }^{22}$ In Barbados, the corresponding proportions were $88 \%, 84 \%$ and $90 \%$ for persons diagnosed with STEMI. These proportions were higher than average medication uptake rates quoted for $10 \mathrm{low} /$ middle-income countries (LMIC) in the WHO-Prevention of Recurrences of Myocardial Infarction and Stroke study ${ }^{23}$ which were aspirin $(81.2 \%), \mathrm{BB}(48.1 \%)$ and statins $(20.8 \%)$.

Barbados, along with eight other Caribbean Community countries, is classified by the World Bank as a high-income country even though its per-capita gross domestic product is one-third that of other similarly classified countries such as the USA. Barbados faces many of the vulnerabilities of being a small island developing state (SIDS). In a study comparing in-hospital trends of revascularisation and prescription of medications at discharge in patients with ACS from high-income (Canada and USA) and LMICs (India, Iran, Pakistan and Tunisia), Shimony et al showed that no patients from 
LMICs were treated by coronary artery bypass graft (CABG) surgery compared with $9.6 \%$ of patients from high-income countries. ${ }^{24}$ Barbados' discharge medication uptake rates mirror those of developed countries but its rates for the costlier intervention services like CABG more closely mirror LMICs likely because it is a SIDS with limited resources and capacity for tertiary care services. The latter was reflected in the non-availability of primary PCI during the study period and may contribute to the relatively high in-hospital case fatality rates. An interventional cardiologist was hired in 2016 and has begun to offer percutaneous angiography to patients non-acutely from 2017. There are currently insufficient resources to offer this modality acutely.

In our study, women consistently had higher prescription proportions on discharge, particularly in the case of BB. Previous international studies had shown slightly higher prescribing among men but more recent analyses from the Acute Coronary Treatment and Interventions Outcomes Network (ACTION) Registry-Get With the Guidelines database obtained from US hospitals showed no significant difference in prescribing between men and women. ${ }^{20}$ The ACTION Registry database is derived from data received on consecutive patients admitted with acute MI, either STEMI or NSTEMI, from more than 450 participating hospitals across the USA. ${ }^{25}$

Limitations on data interpretation should be acknowledged as the analysed sample of patients with unstable angina in particular was relatively small. We did not retrieve the required sample size for the retrospective chart review, thus limiting our power. There may also have been selection bias since several of the records requested could not be found. This is most likely to be a problem for the review of patients with unstable angina where our response rate was only $68 \%$. The BNR is an attempt at full enumeration of all cases of acute MI in the island but, as noted in figure 1, notes are less likely to be retrieved by BNR data abstractors when the person is deceased. These are more likely to be the severe cases, and so our estimates may be reflective of management for less severe acute MI in Barbados.

Data abstraction was being done through the perusal of paper records, which may present several barriers to detecting and accurately documenting medications that have been given. Potential barriers to good data abstraction include poor handwriting of physicians and nurses, poorly assembled notes and omissions of relevant details. As this was retrospective data collection, the data being documented were not specifically written for the purposes of this or any study and thus documentation was often inconsistent.

\section{CONCLUSION}

Studies documenting prescription patterns are not common among SIDS and thus modelled estimates based on assumptions from developed countries are often used as proxies. ${ }^{26}$ We found significant gender disparity in rates of fibrinolytics prescribed in Barbados. Less than $60 \%$ of men and approximately one-third of women receive reperfusion using medical thrombolysis. This analysis is particularly important for Caribbean policymakers since using data from other dissimilar territories can lead to overestimation or underestimation of the effects of interventions. Previous studies from LMICs have had small sample sizes, our findings fill a significant gap in the literature that exists for LMICs and SIDS in particular.

Acknowledgements We acknowledge the support of Dr Amanda Solomon and Dr Zoe Bynoe for their assistance with data collection, data entry and analysis related to the retrospective chart review conducted at the Queen Elizabeth Hospital and the polyclinics throughout Barbados. The data collection and analysis on the retrospective chart review was made possible through a grant from the Campus Research Award Funds, School for Graduate Studies and Research, Cave Hill Campus, University of the West Indies. This research was facilitated by permissions and staff logistical support provided by the Institutional Review Board of the University of the West Indies; the Board of Management and staff of the Queen Elizabeth Hospital; as well as Chief Medical Officer, Permanent Secretary and other staff of the Ministry of Health, Government of Barbados. We acknowledge the data collection work of the team at the Barbados National Registry for Non-Communicable Diseases which is funded by the Ministry of Health, Government of Barbados.

Contributors Study conception and design: NS, AMCR, TAS, IH, NU, JC. Analysis and interpretation of data: NS, AH, MA, AMCR, AH, NU. Drafting of manuscript: NS, AMCR, TAS, JC, NU. Critical revision: NS, AMCR, TAS, JC, MA, IH, AH, NU.

Funding The Barbados National Registry for Non-Communicable Diseases is funded by the Ministry of Health and Wellness, Government of Barbados. The data collection and analysis on the retrospective chart review was made possible through a grant from the Campus Research Award Funds, School for Graduate Studies and Research, Cave Hill Campus, University of the West Indies.

Competing interests None declared.

Patient consent Not required.

Ethics approval University of the West Indies at Cave Hill/Ministry of Health and Wellness, Barbados, and Queen Elizabeth Hospital and the Ministry of Health.

Provenance and peer review Not commissioned; externally peer reviewed.

Data sharing statement Studies using data from the Barbados National Registry for Non-Communicable Diseases are subject to regulations of the Government of Barbados and unrestricted sharing is not allowed. Data can be requested by submitting a request to the University of the West Indies and the Ministry of Health and Wellness.

Open access This is an open access article distributed in accordance with the Creative Commons Attribution Non Commercial (CC BY-NC 4.0) license, which permits others to distribute, remix, adapt, build upon this work non-commercially, and license their derivative works on different terms, provided the original work is properly cited, appropriate credit is given, any changes made indicated, and the use is non-commercial. See: http://creativecommons.org/licenses/by-nc/4.0/.

\section{REFERENCES}

1. Mani KA, Hoo Sang M, Younger-Coleman NO, et al. Ischaemic Heart Disease at the University Hospital of the West Indies: trends in hospital admissions and inpatient mortality rates 2005-2010. West Indian Med J 2014;63:424-30.

2. Bashore TM, Granger CB, Hranitzky P, et al. Current medical diagnosis \& treatment. New York: McGraw-Hill, 2013.

3. Acute myocardial infarction: pre-hospital and in-hospital management. The Task Force on the Management of Acute Myocardial Infarction of the European Society of Cardiology. Eur Heart J 1996;17:43-63.

4. Kramer JM, Newby LK, Chang WC, et al. International variation in the use of evidence-based medicines for acute coronary syndromes. Eur Heart J 2003;24:2133-41.

5. Al-Zakwani I, Zubaid M, Panduranga P, et al. Medication use pattern and predictors of optimal therapy at discharge in 8176 patients with acute coronary syndrome from 6 Middle Eastern countries: data from the gulf registry of acute coronary events. Angiology 2011;62:447-54. 
6. Adams OP, Carter AO. Diabetes and hypertension guidelines and the primary health care practitioner in Barbados: knowledge, attitudes, practices and barriers--a focus group study. BMC Fam Pract 2010;11:96.

7. Graham G. Acute coronary syndromes in women: recent treatment trends and outcomes. Clin Med Insights Cardiol 2016;10:1-10.

8. Rose AM. Understanding the burden of cardiovascular disease in a small island nation with an ageing population. In: University of the West Indies 2017:292.

9. Rose AMC, Maul L, Hambleton IR, et al. 2016 Annual Cardiovascular Disease Report: Barbados National Registry.. George Alleyne Chronic Disease Research Center 2018.

10. Anderson RD, Pepine CJ. Gender differences in the treatment for acute myocardial infarction: bias or biology? Circulation 2007;115:823-6.

11. Shah AS, Griffiths M, Lee KK, et al. High sensitivity cardiac troponin and the under-diagnosis of myocardial infarction in women: prospective cohort study. BMJ 2015;350:g7873.

12. Zhang Z, Fang J, Gillespie C, et al. Age-specific gender differences in in-hospital mortality by type of acute myocardial infarction. Am J Cardiol 2012;109:1097-103.

13. Singh JA, Lu X, Ibrahim S, et al. Trends in and disparities for acute myocardial infarction: an analysis of Medicare claims data from 1992 to 2010. BMC Med 2014;12:190.

14. Yu J, Mehran R, Grinfeld L, et al. Sex-based differences in bleeding and long term adverse events after percutaneous coronary intervention for acute myocardial infarction: three year results from the HORIZONS-AMI trial. Catheter Cardiovasc Interv 2015;85:359-68.

15. Tricoci P, Allen JM, Kramer JM, et al. Scientific evidence underlying the ACC/AHA clinical practice guidelines. JAMA 2009;301:831-41.

16. von Elm E, Altman DG, Egger M, et al. The Strengthening the Reporting of Observational Studies in Epidemiology (STROBE) statement: guidelines for reporting observational studies. J Clin Epidemiol 2008;61:344-9.

17. Rose AM, Hambleton IR, Jeyaseelan SM, et al. Establishing national noncommunicable disease surveillance in a developing country: a model for small island nations. Rev Panam Salud Publica 2016;39:76-85.

18. Zevallos JC, Yarzebski J, Banchs H, et al. Gender disparities in Puerto Ricans hospitalized with an initial acute myocardial infarction: a population-based perspective. $P R$ Health Sci J 2012;31:192-8.

19. Kuhn L, Worrall-Carter L, Ward J, et al. Factors associated with delayed treatment onset for acute myocardial infarction in Victorian emergency departments: a regression tree analysis. Australas Emerg Nurs J 2013;16:160-9.

20. Edmund Anstey D, Li S, Thomas L, et al. Race and sex differences in management and outcomes of patients after ST-Elevation and NonST-Elevation myocardial infarct: results from the NCDR. Clin Cardiol 2016;39:585-95.

21. Bajaj RR, Goodman SG, Yan RT, et al. Treatment and outcomes of patients with suspected acute coronary syndromes in relation to initial diagnostic impressions (insights from the Canadian Global Registry of Acute Coronary Events [GRACE] and Canadian Registry of Acute Coronary Events [CANRACE]). Am J Cardiol 2013;111:202-7.

22. Aliprandi-Costa B, Ranasinghe I, Chow V, et al. Management and outcomes of patients with acute coronary syndromes in Australia and New Zealand, 2000-2007. Med J Aust 2011;195:116-21.

23. Mendis S, Abegunde D, Yusuf S, et al. WHO study on Prevention of REcurrences of Myocardial Infarction and StrokE (WHO-PREMISE). Bull World Health Organ 2005;83:820-9.

24. Shimony A, Grandi SM, Pilote L, et al. Utilization of evidence-based therapy for acute coronary syndrome in high-income and low/ middle-income countries. Am J Cardiol 2014;113:793-7.

25. McNamara RL, Kennedy KF, Cohen DJ, et al. Predicting In-Hospital Mortality in Patients With Acute Myocardial Infarction. J Am Coll Cardiol 2016;68:626-35.

26. Institute for Health Metrics and Evaluation HDN. The World Bank. The Global Burden of Disease: generating evidence, guiding policy Latin America and Caribbean Regional Edition. Seattle, Washington: IHME, 2013. 